\title{
Nivel de satiffacción de las clases online por parte de los estudiantes de Educación Física de Chile en tiempos de pandemia Level of satisfaction of online classes by students of Physical Education of Chile in times of pandemic

\author{
*Elizabeth Flores Ferro, **Fernando Maureira Cid, ***M arcelo Hadweh Briceño, **** Socorro Alonso \\ Gutiérrez Duarte, $* * * * *$ Ángela Silva-Salse, $* * * * * *$ Sebastián Peña-Troncoso, $* * * * *$ Franklin Castillo Retamal, \\ ********Patricia González Flores, ********Francisca Pauvif Cárcamo, **Valentina Bahamondes Acevedo, \\ **Gonzalo ZapataVera, $* * * * * * * *$ J uan Pablo Zavala-Crichton, $* * * * * * * * *$ J uan Maureira Sánchez, \\ $* * * * * * * * * *$ M arcela Brevis-Yéber, $* * * * * * * * * * *$ Carlos Lagos 0 livos \\ *U niversidad Bernardo O'Higgins (Chile), **U niversidad M etropolitana de Ciencias de la Educación (Chile), \\ ***U niversidad SEK (Chile), **** Universidad Pedagógica Nacional del Estado de Chihuahua (M éxico), \\ *****universidad Católica del Maule (Chile), ******U niversidad Austral de Chile (Chile), *******U niversidad Católica \\ Silva Henríquez (Chile), ********U niversidad Andres Bello (Chile), *********U niversidad Central Santiago (Chile), \\ $* * * * * * * * * *$ U niversidad Central Región de Coquimbo (Chile), ***********U niversidad de Tarapacá (Chile)
}

Resumen. Introducción: En Chileel primer caso deCOVID-19 fuediagnosticado el tresdemarzo del 2020 y el díadieciocho del mismo mes el presidente decreto estado de catástrofe, por lo que las clases anivel escolar y universitario pasaron de lapresencialidad alavirtualidad. El presenteestudio tiene como objetivo conocer el nivel de satisfacción de lasclases virtuales delosestudiantes de Educación Física. M etodología: Cuantitativa, no experimental, transversal. Lamuestraestuvo constituidapor 542 al umnos deEducación Físicadediferentes casas deestudio deChile. Se adaptó y validó la escalaSatisfacción clases online. Resultados: existen diferenciassignificativas comparando las asignaturasteóricas y prácticas, siendo mejor valoradas las cátedrasteóricas. También seencontraron diferencias por sexo, donde los varones poseen una percepción másnegativa sobre las clases virtualesy al comparar por curso, los alumnosde primer año poseen unapercepción más positiva sobrelasclases virtuales en relación con los cursos superiores. Conclusiones: existe una resistencia por parte de los alumnos a las clases virtuales en la Educación Física, pues, si bien se encontraron diferenciassignificativas entre las asignaturasteóricasy prácticas, losvaloressiempreestuvieron al rededor de 3 en unaescala de 1 a5. Se hacen necesariasfuturasinvestigaciones con otras variables como actividad física, niveles de estrés y estrategiasparalaenseñanza de laEducación Física virtual.

Palabrasclaves: Educación física, clasesvirtuales, pandemia.

\begin{abstract}
Introduction: In Chile the first case of COVID-19 was diagnosed on M arch 3, 2020 and on the eighteenth of the same month the president decreed state of catastrophe, so that classesat school and university level went from the presence to the virtual ity. The present study aims to know the level of satisfaction of the virtual classes of the students of Physical Education. M ethodology: Q uantitative, non experimental, transversal. The sample consisted of 542 students of Physical Education from different study houses in Chile. The Satisfaction scale online classeswas adapted and validated. Results: there are significant differencescomparing theoretical and practical subjects, with theoretical chairs being better valued. Differences were also found by sex, where males have a more negative perception about virtual classes and when comparing by course, freshmen have amore positive perception about virtual classes in relation to higher courses. Conclusions: there isa resistance on the part of studentsto virtual classes in Physical Education, because, although significant differences were found between the theoretical and practical subjects, values were al ways around 3 on a scal e of 1 to 5 . Future research with other variables such as physical activity, stress levels and strategies for the teaching ofV irtual Physical Education arenecessary.
\end{abstract}

Keywords: Physical education, virtual classes, pandemic.

\section{Introducción}

Una reciente enfermedad conocida como Coronavirus (COVID-19) está azotando actualmente a la humanidad, circula alrededor del mundo desde el pasado mes de diciembre de 2019, y es causada por una nueva sepade coronavirus denominadaSARSC oV-2, que se propaga de persona a persona con un aumento des-

Fecha recepción: 26-08-20. Fecha de aceptación: 03-12-20 Elizabeth Flores Ferro

prof.elizabeth.flores@gmail.com medido de contagios, lo que pone en riesgo la salud de los ciudadanos de diversos pueblos del planeta. La O rganización M undial de la Salud (O MS) define a esta enfermedad como una gran familia de virus capaz de propagarse en animales y seres humanos, en este último provoca infecciones respiratorias que van desde un resfriado común hasta un Síndrome Respiratorio Agudo Severo (O MS, 2020).

El incremento acelerado de contagios obligó a la O rganización Mundial de la Salud (OMS) a declarar la epidemia como una Emergencia de Salud Pública de Importancia Internacional el 30 de enero de 2020, y 
para el 11 de marzo del mismo año, se le da el carácter de pandemia; al mismo tiempo, emite una serie de recomendaciones y medidas con el propósito de contener el número de contagios y afectaciones por parte del citado virus, entre las que destacan aumentar las condiciones de higiene y disminuir al máximo las posibilida des de contacto entrelas personas, ocasionando con ello, un necesario distanciamiento social.

Ducoing (2020) señala que el COVID-19 ha obligado a la mayoría de los gobiernos a cerrar indefinida mente las instituciones de enseñanza para así evitar la propagación del virus. Para dar continuidad a los procesos de formación y de enseñanza aprendizaje, la UNESCO (2020) recomienda el uso de las tecnologías de la información y comunicación (TIC) como la herra mienta principal para el desarrollo de las labores educa tivas en todos los niveles.

En Chile, desde el mes de marzo se declara la pandemia a nivel nacional y a partir de ello se generan una serie de medidas para los niveles de primaria y secundaria (Gobierno de Chile, 2020). El día 15 de marzo, se plantean las orientaciones del Ministerio de Educación (MINEDUC) y se empieza a hablar de aprendizaje remoto. El 25 de marzo se confirma la suspensión de las clases medida que se ha mantenido hasta el día de hoy (17 de agosto de 2020). Para la enseñanza superior, se establece el Protocolo $\mathrm{N}^{\circ} 2$ el día 13 de marzo (MINEDUC, 2020a) con el objetivo de disminuir los riesgos de propagación por el medio del aisla miento de casos y/ o comunidades. Posteriormente, se establece un plan de acción que considera plataformas online, fondos para el desarrollo y fortalecimiento de proyectos online y difusión de buenas prácticas en esta modalidad.

En abril la Subsecretaria de la Educación Superior (MINEDUC, 2020b) oficia las instituciones para saber las medidas para poder continuar con los procesos formativos de los estudiantes. Por lo que, se lleva a cabo un plan de fiscalización a través de la Superintendencia de Educación Superior con 45 instituciones, pero todavía se encuentra en análisis, porque no se han publicado los resultados (Superintendencia de la Educación Superior, 2020).

En relación con las investigaciones de las clases a distancia, se puede mencionar a Fujimoto (2020) quien enfatiza que la educación virtual recurre a herramientas tecnológicas que facilitan el proceso educativo: banda ancha, audio y video compatibles con la red de conexiones que utiliza, personal con competencias tecnológicas y pedagógicas, contenidos virtuales, etc. Por su parte, A tamara (2020) hace referencia que en la educa ción presencial el profesor adquiere la responsabilidad de desarrollar y explicar los contenidos, mientras que, en la modalidad virtual, el alumno asume el papel de protagonista de manera indiscutible, y el profesor cambia su rol como un orientador, guía y facilitador del proceso de enseñanza-aprendizaje.

Aunque la modalidad de educación virtual no es reciente, el repentino cambio de paradigma educativo exige a los actores principales del proceso de enseñanza y aprendizaje, afrontar una serie de transformaciones que impone la nueva escuela. De manera particular, los académicos universitarios se han enfrentado a diversas plataformas de educación virtual, y probablemente también se encuentren experimentado estrategias que demanda la educación a distancia, considerando que en las clases presenciales se podía interactuar entre el profesor y estudiante (Ruíz, 2020). En este sentido, García (2011) propone en su teoría denominada diálogo didáctico mediado de educación virtual, la existencia de comunicación y diálogo permanente entre profesores y alumnos, donde el estudiante no se limite a responder, sino a preguntar o iniciar él mismo la interacción con el maestro.

Es preciso señalar que durante las últimas décadas se han desarrollado experiencias investigativas en diversas instituciones de educación superior, implementando nuevas modalidades de estudios, las cuales, involucran el uso de las tecnologías de la información y comunica ción (TIC), trasformando la enseñanza presencial a tra vés del uso de plataformas 0 entornos virtuales de enseñanza aprendizaje (Fernández-Pascual, Ferrer-Cascales \& Reig-Ferrer, 2013), resaltando la importancia de las TIC en la formación docente y del aprendizaje perma nente (M onguillot, Guitert \& González, 2013). Por otra parte, las instituciones de educación superior han realizados esfuerzos para entregar servicios complementa rios, lo que implica que el proceso formativo tenga presente distintas aristas complejas que influyen en la satisfacción estudiantil (Fayos, González-Gallarza, Servera $\&$ Arteaga, 2011).

En este sentido, un estudio realizado por Zambrano, M edinay García(2010) aplicaron dos cuestionarios, uno para el profesorado y otro para los alumnos, a dos cursos del Programa deAdministración de Empresas de la Fundación U niversitaria Panamericana. Los resultados evidencian el cambio del profesor tutor principalmente como un facilitador y consejero, destacando su compromiso, entusiasmo, coordinación y apoyo para el buen desarrollo del curso; su papel como guía fue promover 
el desarrollo integral de los estudiantes. Con respecto a los alumnos, se encontró que estos asumieron un rol más activo y dinámico a partir de la interacción con los contenidos, empleando para su estudio una estructura abierta donde el espacio no es sinónimo de lugar fijo, con destacado trabajo de campo para la preparación de los temas y contenidos; así mismo se debe resaltar la motivación de los discentes, además de la disciplina y confianza en las labores desarrolladas al trabajar en equipo.

Palominos-Belmar, Q uezada-Llanca, O sorio-Rubio, Torres-O rtega y Lippi-Valenzuela (2016) evaluaron a 2.086 estudiantes de una universidad pública de Chile. Los resultados obtenidos en la correlación de Pearson entre los siete factores del cuestionario para medir la satisfacción de usuario del servicio formación educativa universitaria, arrojó en términos generales una correlación positiva, entre niveles baja y media según los factores comparados. A nivel individual de los factores, se encontraron diferencias significativas que resaltan las tres relaciones de nivel media del factor de satisfacción general del usuario con la satisfacción con el servicio docente $(p=.624)$, con la evaluación de los resultados $(p=.631)$ y con lareputación de lainstitución $(p=.668)$. También se encontró relación media entre los factores de la evaluación de los resultados y la reputación de la institución $(p=.609)$. Se concluye que los estudiantes de la institución pública del estudio perciben una eva luación de los resultados satiffactoria de su proceso de aprendizaje y que reflexionen sobre el beneficio adquirido al estudiar en una universidad pública.

En una investigación realizada por Cabero, Llorente y Puentes (2010) se abordaron cursos de introducción a la filosofía y física introductoria con 284 estudiantes de la Pontificia U niversidad Católica M adre y Maestra de la República Dominicana (PUCM M) en modalidad blearning Acerca de los instrumentos de recolección de datos, los autores señalan que aplicaron el cuestionario de satisfacción de alumnos universitarios (CASAUF), una encuesta en línea a los estudiantes y una entrevista electrónica a los profesores-tutores. En relación con los resultados principales, la media más alta corresponde a ladimensión aspectos rel acionados con el profesor/ tutor online (3.25 \pm .15$)$ y la más baja a los aspectos relacionados con la comunicación entre profesor-estudiante y pares $(2.95 \pm .21)$ considerando que $84 \%$ del total de la muestra arrojó una puntuación superior a tres(3), por lo cual, los autores consideran que los alumnos poseen una satisfacción media de los estudiantes con la experiencia b-learning.

Si bien, existen experiencias innovadoras y exitosas en procesos de formación y de enseñanza aprendizaje a través de medios virtuales como por ejemplo el trabajo realizado por Dong y Yu (2020) investigaron la implementación de la realidad virtual del tiro al arco en modelos 3D para el ámbito deportivo con el objetivo de satisfacer la necesidad de la educación física. Los autores concluyeron que con la ayuda de la computación y la tecnología virtual se puede generar y heredar la cultura deportiva, mejorando la comprensión de los estudiantes hacia estos deportes tradicionales. Por su parte, Bopp y Stellefson (2020) investigaron desafíos y oportunidades del uso de las redes sociales para mejorar la alfabetización física en jóvenes. Los autores concluyeron que, si los educadores físicos utilizan diferentes estrategias en línea, los estudiantes se beneficiaran a tra vés de las redes sociales populares para promover la práctica de actividad física. Por otro lado, también hay evidencias que no siempre las clases virtuales han sido beneficiosas, como en el estudio de Guest, Rohde, Selvanathan y Soesmanto (2018) donde evaluaron 2653 cursos desde el año 2011 para estudiar el impacto de la transición de clases presenciales al aprendizaje en línea, llegando a la conclusión a que existe un leve rechazo a las clases virtuales. Sin embargo, dicha modalidad se ha convertido en un elemento más, que no llega a modificar de fondo las prácticas educativas (A dell y Castañeda, 2012), pues no se trata de incluir la tecnología nada más por incluirla, sino «que real mente sea una disrupción que motive cambios profundos en las prácticas pedagógicas cotidianas» (Barrón, 2020:70).

Con base en lo expuesto, surge el interés de indagar acerca del nivel de satisfacción de los estudiantes de Educación Física(EF) de Chile, quellevaron clasesonline durante la pandemia ocasionada por el Covid-19.

\section{Método}

\section{Muestra}

De tipo no aleatoria intencionada. Estuvo constituidapor 542 estudiantes de Pedagogía en Educación Física de ocho instituciones de educación superior de Chile. Del total, 183 son de sexo femenino (33.8\%) y 359 de sexo masculino (66.2\%). 101 estudiantes cursaban primer año de la carrera (18.6\%), 132 segundo año $(24.4 \%), 115$ tercer año $(21.2 \%), 115$ cuarto año $(21.2 \%)$ y 79 quinto año (14.6\%).

\section{Instrumento}

Se utilizó una adaptación de la escala de Satisfacción de Recio y Cabero (2005). De los 39 ítems originales, 
se utilizaron las 13 preguntas que exploran la satisfacción de los alumnos sobre las clases, contenidos, evaluar ciones e interacción (las otras 26 preguntas apuntan a contenidos diferentes). La escala es de tipo Likert que va desde $1=$ totalmente en desacuerdo hasta $5=$ totalmente de acuerdo. Los alumnos contestaron dos veces las 13 preguntas, la primera orientada a las clases virtuales teóricas (cátedras como currículum, Anatomía, Biomecánica, etc.) y la segunda para las clases virtuales prácticas (asignaturas deportivas).

En la presente muestra, la escala de Satisfacción para asignaturas teóricas online presenta un nivel de confiabilidad de á $=.896$. En relación con su validez se obtuvo un $\mathrm{KMO}=.937$ y una prueba de esfericidad de Bartlett de $p=.000$, con estos antecedentes de procedió a aplicar aplicó un análisis factorial exploratorio que entregó la existencia de un componente que explica el $45.24 \%$ de la varianza total.

La escala de Satisfacción para asignaturas prácticas online presenta un nivel de confiabilidad de á $=.931$. En relación con su validez se obtuvo un $\mathrm{KMO}=.951$ y una prueba de esfericidad de Bartlett de $p=.000$. El análisis factorial exploratorio entregó la existencia dos componentes que explican el $63.63 \%$ de la varianza total.

\section{Procedimiento}

La encuesta se aplicó en estudiantes de Pedagogía en Educación Física de Chile vía correo electrónico durante el mes de junio 2020. En el correo se incluyó una breve descripción de la investigación con el objetivo y un enlace utilizando la herramienta Formulario de Google. En relación con el consentimiento informado, también fue online dondelos sujetos debían leer y finalmente aceptar para poder contestar la encuesta.

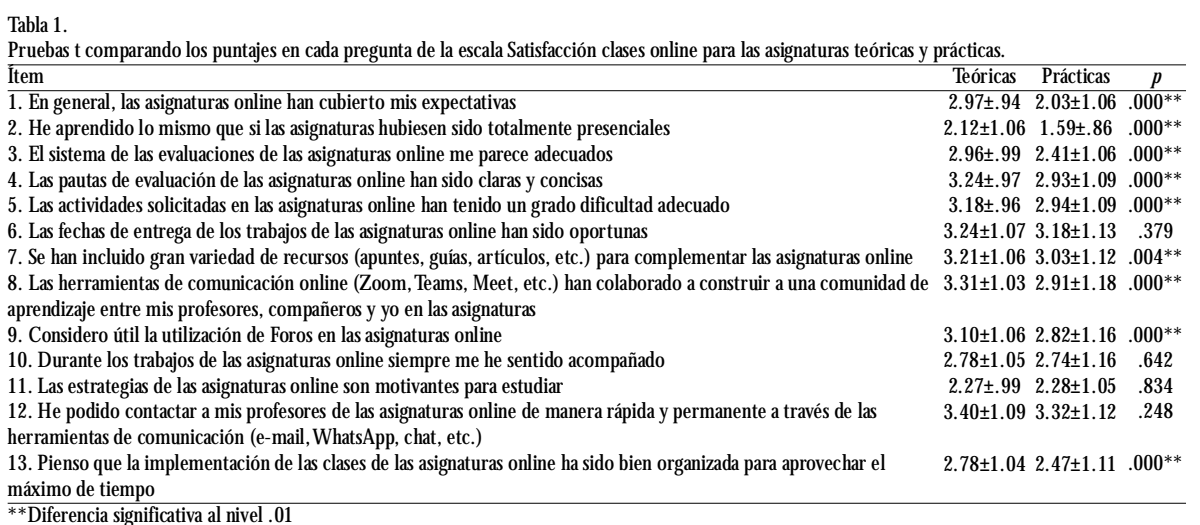
2. He aprendido lo mismo que si las asignaturas hubiesen sido totalmente presenciales

3. El sistema de las evaluaciones de las asignaturas online me parece adecuados

5. Las actividades solicitadas en las asignaturas online han tenido un grado dificultad adecuado

. D urante los trabajos de las asignaturas online siempre me he sentido acompañado

.

as online ha sido bien organizada para aprovechar máximo de tiempo

** Diferencia significativa al nivel .01

\section{Análisis de datos}

Se utilizó el programa estadístico IBM SPSS 25.0 para Windows. Se trabajó con estadística descriptiva como medias, desviaciones estándar y frecuencias. Las pruebas de normalidad entregaron valores $p>.05$ en todas las variables, razón por la cual, se aplicó análisis inferenciales como pruebas t para muestras independientes para comparar entre asignaturas teóricas y prácticas, y para comparar según sexo, pruebasAN OVA para comparar entre cursos y pruebas de Correlaciones de
En la figura 1 y 2 se observan los puntajes y las pruebas t para muestras independientes comparando los puntajes de cada pregunta de la escala Satisfacción cla ses online entre damas y varones. En relación con las asignaturas teóricas el ítem 2-H e aprendido lo mismo que si las asignaturas hubiesen sido totalmente presenciales, el ítem12-H e podido contactar a mis profesores de las asignaturas online de manera rápida y permanente a través de las herramientas de comunicación (e mail, WhatsApp, chat, etc.) y el ítem 13-Pienso que la implementación de las clases de las 
asignaturas online ha sido bien organizada para aprovechar el máximo de tiempo presentan puntajes más altos en las da mas. En relación con asignaturas prácticas el ítem 5- Las actividades solicitadas en las asignaturas online han tenido un grado dificultad adecuado, el ítem 7-Se han incluido gran variedad de recursos (apuntes, guías, artículos, etc.) para complementar las asignaturas online, el ítem 8-Las herramientas de comunicación online (Zoom, Teams, M eet, etc.) han colaborado a construir a una comunidad de aprendizaje entre mis profesores, compañeros y yo en las asignaturas y el ítem 13Pienso que la implementación de las clases de las asignaturas online ha sido bien organizada para aprovechar el máximo de tiempo nuevamente presentan puntajes más altos en las damas.

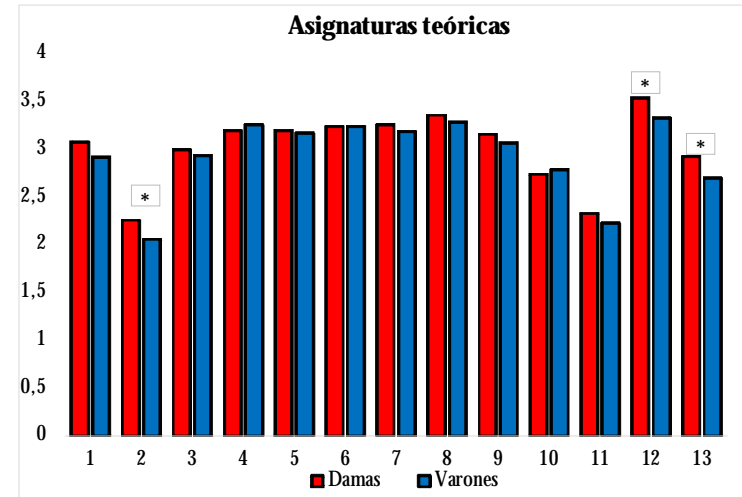

Figura 1. Puntajes y pruebas t comparando los puntajes de cada pregunta de la escala Satisfacción cl ases online para las asignaturas teóricas según el sexo de la muestra.

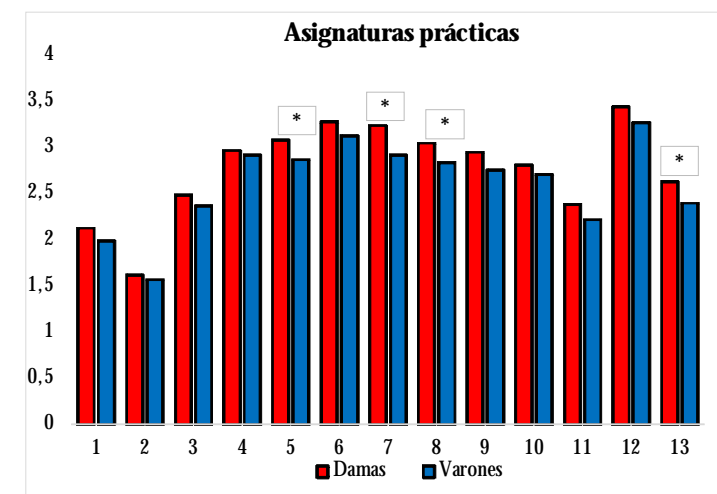

Figura 2. Puntajes y pruebas t comparando los puntajes de cada pregunta de la escala Satisfacción clases online para las asignaturas prácticas según el sexo de la muestra

En la tabla 2 y 3 se muestran las pruebasANOVA y los post-hoc deTukey comparando los puntajes de cada ítem de la escala Satisfacción clases online según cursos de la muestra. En relación con las asignaturas teóricas el ítem 2-He aprendido lo mismo que si las asignaturas hubiesen sido totalmente presenciales, el ítem 5-Las actividades solicitadas en las asignaturas online han tenido un grado dificultad adecuado, el ítem 7-Se han incluido gran variedad de recursos (apuntes, guías, artículos, etc.) para complementar las asignaturas online y el ítem 11-Las estrategias de las asignaturas online son motivantes para estudiar presentan diferen- cias por cursos con puntaje más al tos en primer año en cuatro de estos ítems. En relación con las asignaturas prácticas el ítem 1-En general, las asignaturas online han cubierto mis expectativas y el ítem 2-He aprendido lo mismo que si las asignaturas hubiesen sido totalmente presenciales presentan diferencias por curso con puntajes más alto en quinto año.

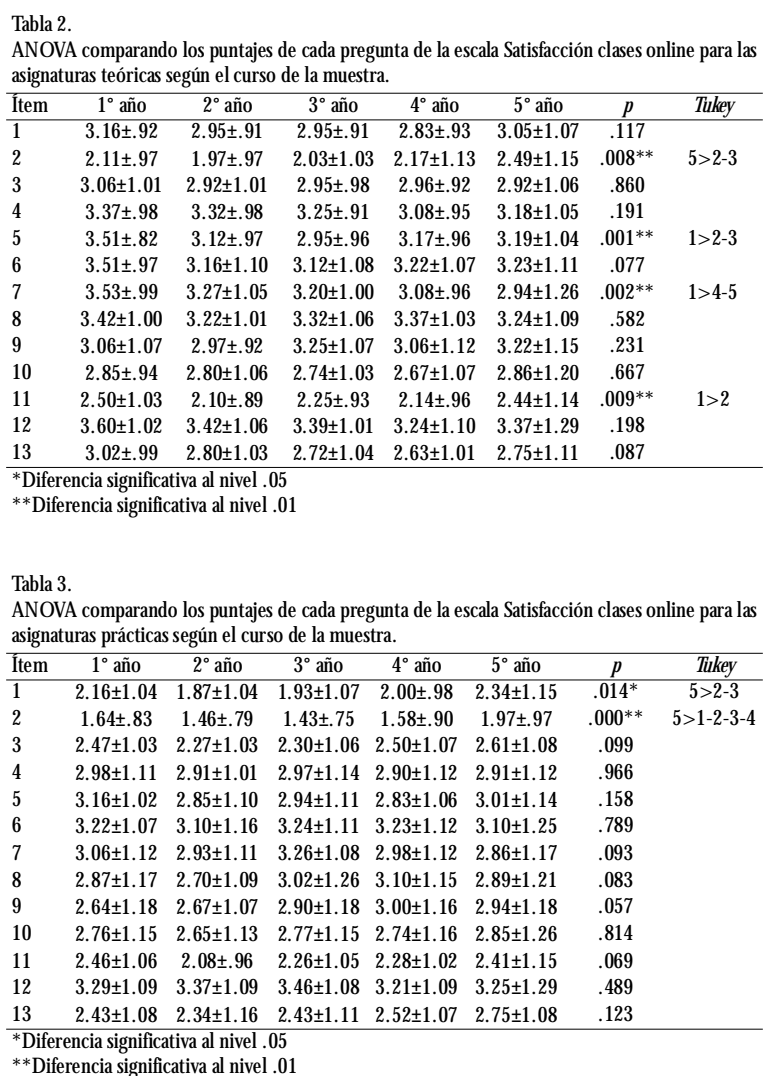

Del total de la muestra, 21 sujetos (3.8\%) manifiesta tener un nivel bajo de conectividad a internet (valores 1 y 2), 344 sujetos (63.5\%) dice tener un nivel medio de conectividad (valores 3, 4 y 5) y 177 sujetos $(32.6 \%)$ manifiesta tener un nivel alto de conectividad (valores 6 y 7). En la tabla 4 se observan las correlaciones entre los niveles de conectividad y las puntuaciones asignadas a cada pregunta de la escala Satisfacción clases online. Es posible notar que todos los ítems presentan

Tabla 4.

Correlaciones de Pearson entre los niveles de conectividad y los puntajes de cada pregunta de la escala Satisfacción clases online en las asignaturas teóricas y prácticas del total de la muestra.

\begin{tabular}{lccc}
\multicolumn{2}{c}{ escala Satisfacción clases online en las asignaturas teoricas y practicas del total de la muestra. } \\
\hline & Asignaturas té́ricas & \multicolumn{2}{c}{ Asignaturas prácticas } \\
\hline Item & Conectividad & Item & Conectividad \\
\hline 1 & $.259^{* *}$ & 1 & $.219^{* *}$ \\
2 & $.236^{* *}$ & 2 & $.191^{* *}$ \\
3 & $.196^{* *}$ & 3 & $.201^{* *}$ \\
4 & $.242^{* *}$ & 4 & $.183^{* *}$ \\
5 & $.227^{* *}$ & 5 & $.217^{* *}$ \\
6 & $.252^{* *}$ & 6 & $.190^{* *}$ \\
7 & $.154^{* *}$ & 7 & $.107^{* *}$ \\
8 & $.230^{* *}$ & 8 & $.195^{* *}$ \\
9 & $.126^{* *}$ & 9 & $.154^{* *}$ \\
10 & $.252^{* *}$ & 10 & $.255^{* *}$ \\
11 & $.215^{* *}$ & 11 & $.184^{* *}$ \\
12 & $.163^{* *}$ & 12 & $.154^{* *}$ \\
13 & $.170^{* *}$ & 13 & $.186^{* *}$ \\
\hline$*$ Correlación significativa al nivel .05 & &
\end{tabular}


correlaciones significativas con la conectividad ainternet, aunque estas son de nivel bajo con $r<.259$. La magnitud de correlación fue basada en la siguiente escala: trivial (<.10), pequeña (.10 - .29), moderada (.30 - .49), alta (.50 - .69), muy alta (.70 - .89), casi perfecta (>.90), y perfecta ( $r=1)$ (Rovai, Baker, \& Ponton, 2014).

\section{Discusiones}

Dentro de los principales hallazgos de este estudio, se puede mencionar que, al comparar los cursos y las cátedras teóricas, se puede observar que los estudiantes de primer año perciben de mejor manera las clases virtuales de la Licenciatura de Educación Física, estos resultadoscoinciden con el estudio de Cea,V éliz, A ravena y Maureira (2013) donde también identificaron que mientras los participantes del estudio cursaban los niveles iniciales de su carrera universitaria, la percepción de los alumnos eran más positivas. No obstante, en el reactivo dos que se refiere a $\mathrm{H}$ e aprendido 10 mismo que si las asignaturas hubiesen sido totalmente presenciales, donde los alumnos de quinto año presentan los puntajes más altos en comparación a los cursos inferiores. Ello puede estar dado porque los alumnos están más cerca de titularse de la carrera y en sus respectivos procesos de práctica profesional, por lo tanto, realizan reflexiones más profundas sobre su quehacer pedagógico (Tallaferro, 2005). Lo mismo ocurre al comparar los cursos, pero en las asignaturas prácticas, donde los al umnos de quinto año perciben de mejor manera cuando responden a los reactivos En general, las asignaturas online han cubierto mis expectativas y H eaprendido lo mismo que si las asignaturas hubiesen sido totalmente presenciales, sin embargo, ambos promedios no superan el 2.5 de la escala de uno a cinco. Por último, se encontraron correlaciones leves en todos los reactivos de la escala Satisfacción clases online con los niveles de conectividad, ya que el alumnado necesita ciertas condiciones para poder aprender virtualmente (Fujimoto, 2020), por ende, su nivel de satisfacción se verá influenciada por sus recursos tecnológicos.

En la literatura consultada, no existen muchas investigaciones indagando sobre la virtualidad en la forma ción inicial docente en Educación Física, ya que si bien existe una tendencia de enseñar la disciplina desde la acción, es importante hacer hincapié, que el conocimiento desde una perspectiva sistémica y compleja (Sergio, 2019; M oreno,Toro y Gómez, 2019) no se puede fragmentar (Peña, Toro, Cárcamo, HernándezM osqueira y Cresp, 2021), pero si es posible profundizar 0 ampliar cada dimensión, ya sea procedimental, conceptual 0 actitudinal.

Desde lo anterior, la virtualidad de las clases a provocado un quiebre paradigmático para muchos docentes que aún visualizan la motricidad escolar desde una visión biomédica y mecánica, basada principalmente en la tecnificación del aprendizaje (Cardozo, Velasco, Domínguez, \& Reyes, 2019) el cual es evaluado desde el rendimiento delacondición física(M oreno \& M edina, 2012; Zaragozá \& M artínez, 2018). En esta línea, es importante conocer la satisfacción de los estudiantes, los cuales se transformarán en futuros docentes, y son ellos los que pueden generar la creación de nuevas didácticas en la EF tanto en la formación del profesora do como para los alumnos de a nivel escolar.

No obstante, el contexto de la pandemia a nivel mundial, pueden generar nuevas investigaciones similares a esta no tan sólo percibiendo la implementación de las clases online, si no también la creación de nuevas didácticas en la EF tanto en la formación del profesorado como para los alumnos a nivel escolar, con ello a su vez mejorar los niveles de retención y disminuir los porcentajes de deserción, ya que, las investigaciones indican que los motivos se encuentran relacionadas con la gestión de calidad en la universidades ( M atheu, Ruff, Ruiz, Benites \& M orong, 2018; Ruff, Ruiz, Matheu, Argomedo \& Benites, 2017).

\section{Conclusiones}

Sin duda, es un desafío para los docentes aprender a utilizar las nuevas herramientas tecnológicas para mejorar las experiencias de aprendizaje de los educandos, pero tampoco se puede pensar que la Educación y la Educación Física vuelva a ser la misma después de esta transformación digital. Los avances de la computación han repercutido en diferentes áreas de conocimiento y pensar hace al gunos años en una Educación Física virtual era totalmente inimaginable. Los procesos de enseñanza y aprendizaje se han tenido que adecuar a una moda lidad virtual que tanto el profesor y el educando no estaban preparados, pues no hubo tiempo de reflexionar y preparar un plan de acción para las diferentes áreas de conocimiento y didácticas que siempre estuvo pensada desde la experiencia práctica. Los resultados de esta investigación dan cuenta de una resistencia de los estudiantes de Licenciatura de Educación Física hacia un programa virtual, sobre todo en las asignaturas deportivas, ya que ellos perciben que estas cátedras no han cumplido sus expectativas y que no han aprendido lo mismo que si hubiesen sido las clases presenciales. Por 
otro lado, también se puede mencionar que, al compa rar los resultados entre las asignaturas teóricas y prácticas, en su mayoría las mejores puntuaciones son en los ramos teóricos, obteniendo diferencias significativas en nueve de los trece reactivos de la escala a favor de las asignaturas teóricas. En relación con la comparación por sexo de la muestra, se puede observar que los varones cal ifican de forma más negativa a la implementación de las clases virtual es tanto de las cátedras teóricas como prácticas.

Dentro de las proyecciones de esta investigación, resulta interesante realizarlo en otros países considerando otras variables de estudio, como niveles de estrés, nivel de manejo de plataformas tecnológicas, niveles de actividad física, entre otros, para comprender cuales son losfactoresque están influyendo en el proceso de aprendizaje virtual de nuestros educandos. Finalmente, dentro de las limitaciones de esta investigación se puede mencionar el diseño y tipo de investigación, pues se podría revisar diferentes estrategias para enseñar asignaturas prácticas para mejorar la satisfacción del estudiantado sobre sus experiencias de aprendizajes virtuales.

\section{Referencias}

Adell, J. y L. Castañeda. (2012). Tecnologías emergentes, ¿Pedagogías emergentes? En J. Hernández, M. Penessi, D. Sobrino y A. Vázquez (Coord.) Tendencias emergentes en educación conTIC (pp. 13-32). Barcelona: Asociación Espiral, Educación y Tecnología.

Atamara, T. (2020). La educación virtual en tiempos de pandemia. Disponible en: http:/ / udep. edu. pe/ hoy/ 2020/ la-educacion-virtual-en-tiempos-depandemial

Barrón, M. (2020). La educación en línea. Transicionesy disrupciones. En H. CasanovaCardiel (Coord.), Educación y pandemia: una visión académica (pp. 66-74). Ciudad de México: Universidad Nacional Autónoma de México, Instituto de Investigaciones sobre la Universidad y la Educación.

Bopp, T., \& Stellefson, M. (2020). Practical and Ethical Considerations for Schools U sing Social Media to Promote Physical Literacy in Youth. International Journal of Environmental Research and Public H ealth, 17(4), 1225.

Cabero, J., Llorente, M. \& Puentes, Á. (2010). La satisfacción de los estudiantes en red en la formación semipresencial. Revista Comunicar, 18(35), 149-157.

Cardozo, J., Velasco, D., Domínguez, S., \& Reyes, J. (2019). ¿Q ué se investiga en formación docente en educación físicay en recreación? (W hat is researched in teacher training in physical education and recreation?). Retos: nuevas tendencias en educación física, deportey recreación, (36), 3-8.

Cea, S., Veliz, C., Aravena, C. y Maureira, F. (2014). Percepción de los estudiantes de educación física de la UISEK de Chile hacia sus profesores. Cuadernos de Psicología del Deporte, 14 (1), 23-28.

Dong, W. , \&Yu, J. (2020, July). Explore an Evolution of Physical Education Based on Virtual Reality Lab for Traditional Ethnic M inorities' Sports. In International Conference on Human-Computer Interaction (pp. 394401). Springer, Cham.

Ducoing, P. (2020). U na expresión de la desigualdad en educación básica durante la emergencia sanitaria: el caso de una alumna. En H. Casanova Cardiel (Coord.), Educación y pandemia: una visión académica (pp. 55-64). Ciudad de M éxico: Universidad Nacional Autónoma de M éxico, Instituto de Investigaciones sobre la U niversidad y la Educación.

Fayos, T., González-Gallarza, M., Servera, D. \& Arteaga, F. (2011). Análisis y evaluación del servicio de formación universitaria: implicaciones para el marketing estratégico de las universidades. Revista de Investigación en Educación, 9(2), 133-152.

Fernández-Pascual, M., Ferrer-Cascales, R. \& ReigFerrer, A. (2013). Entornos virtuales: predicción de la satisfacción en contexto universitario. Pixel-Bit. Revista de M edios y Educación, 1, 167-181.

Fujimoto, G. (2020). El proceso educativo de la primera infancia en época del coronavirus COVID-19. Informe para las Comisiones A cadémicas de la Red Hemisférica de Parlamentarios y Exparlamentarios por la Primera Infancia. (Documento No Publicado).

García, L. (2011). Perspectivas teóricas de la educación a distancia y virtual. Revista Española de la Pedagogía, 62(249), 255-272.

Gobierno de Chile (2020). Coronavirus. Disponible en: https:/ / www.gob.cl/ coronavirus/

Guest, R., Rohde, N., Selvanathan, S. \& Soesmanto, T. (2018). Student satisfaction and online teaching. Assessment \& Evaluation in Higher Education, 43(7), 1084-1093.

M atheu, A., Ruff, C., Ruiz, M., Benites, L., \& Morong, G. (2018). M odelo de predicción de la deserción estudiantil de primer año en la U niversidad Bernardo 0 ' Higgins. Educaçao e Pesquisa: R evista da Faculdadede Educação da U niversidade de São Paul 0, 44, 1-23

Maureira, F. (2017). Estadística para educación física. Ma 
drid: Bubok Publishing

Maureira, F. \& Flores, E. (2018). Manual de investigación cuantitativa para estudiantes de educación física. M adrid: Bubok Publishing.

Ministerio de Educación (2020a). Protocolo N ²: Coronavirus COVID-19 en instituciones de educación superior. Disponible en https: / / www. mineduc.cl/ wpcontent/ uploads/ sites/ 19/2020/03/ ProtocoloCoronavirus IES.pdf

Ministerio deEducación (2020b). Plan deacción MIN EDUC para Instituciones de Educación Superior. Disponible en https:/ / educacionsuperior.mineduc.cl/ wp-content/ uploads/ sites/49/2020/ 03/ PLAN-DE-ACCIONEDUCACION-SUPERIOR-COVID-19-1.pdf

M onguillot, M., Guitert, M . \& González, C. (2013). El trabajo colaborativo virtual: herramienta de formación del profesorado de educación física. Retos, 24, 24-27.

Moreno, A. \& M edina, J. M. (2012). Número Especial: Escuela, educación física y transformación social. Es tudios pedagógicos (Valdivia), 38 (ESPECIAL), 7-11.

Moreno, A., Toro, S. \& Gómez, F. (2019). Formación inicial de maestros de educación física: conectando un quehacer pedagógico decolonial con la intervención social, política e insurgente del espacio público. Retos, 37, 605-612.

OMS, (2020). Declaración sobre la cuarta reunión del Comité de Emergencia del Reglamento Sanitario Internacional (2005) sobre el brote de la enfermedad por el coronavirus de 2019 (COVID-19). Recuperado de https:/ / www. who.int/ es/ news-room/ statements

Palominos-Belmar, P., Q uezada-Llanca, L., O sorio-Rubio, C., Torres-O rtega, J. \& Lippi-Valenzuela, L. (2016). Calidad de los servicios educativos según los estudiantes de una universidad pública en Chile, Revista Iberoamericana de Educación Superior, 18 (7), 130142.

Peña, S. , Toro, S. , Cárcamo, J., Hernández-M osqueira, C., \& Cresp, M. (2021). Lafragmentación del conocer en educación física. Retos, 39, 231-237.

Recio, M. \& Cabero, J. (2005). Enfoques de aprendiza je, rendimiento académico y satisfacción de los alumnos en formación en entornos virtuales. Revista de Mediosy Educación, 25, 93-115.

Rovai, A., Baker, J., \& Ponton, M. (2014). Social science research design and statistics: a practitioner's guide to research methods and IBM SPSS analysis. Chesapeake (Va.): Watertree Press.

Ruff, C., Ruiz, M. M atheu A., Argomedo, J. \& Benites,
L. (2017). Gestión de la investigación e impacto en la calidad en la Universidad Bernardo 0 'higgins (UBO). Conocimiento para el Desarrollo, 8(1), 81-88.

Ruiz, E. (2020). La práctica docente universitaria en ambientes de educación a distancia. Tensiones y experiencias de cambio. En H. Casanova Cardiel (Coord.), Educación y pandemia: una visión académica (pp. 109-113). Ciudad de México: U niversidad Na cional Autónoma de M éxico, Instituto de Investiga ciones sobre la Universidad y la Educación.

Sérgio, M. (2019). Da Ciência à Transcendência: Epistemologia da Motricidade Humana. Leya.

Superintendencia de Educación Superior (2020). Superintendencia de Educación Superior aplicará plan especial de fiscalización a 45 instituciones por servicios entregados durante la emergencia. Disponible en: https: / / www. sesuperior.cl/ superintendencia-de-educacionsuperior-aplicara-plan-especial-de-fiscalizacion-a-45instituciones-por-servicios-entregados-durante-laemergencial

Tallaferro, D. (2005). La formación para la práctica reflexivaen las prácticas profesional es docentes. Educere, 10(33), 269-273.

UNESCO (2020). El coronavirus COVID-19 y la educación superior: impacto y recomendaciones. Disponible en: http:/ / www. iesalc. unesco. org/ 2020/ 04/ 02/ elcoronavirus-covid-19-y-lareducacion-superior-impacto-y-recomendaciones/ \&gt

Zambrano, W., Medina, V. \& García, V. (2010). Nuevo rol del profesor y el estudiante en la educación virtual. Dialéctica Revista de Investigación/ Educación y Sociedad, 26, 51-61.

Zaragozá, J. \& Martínez, A. (2018). Conectando teoría y práctica profesional en el Grado en Ciencias de la Actividad Física y el Deporte. Revista Española de Educación Física y Deportes, 420, 39-57.

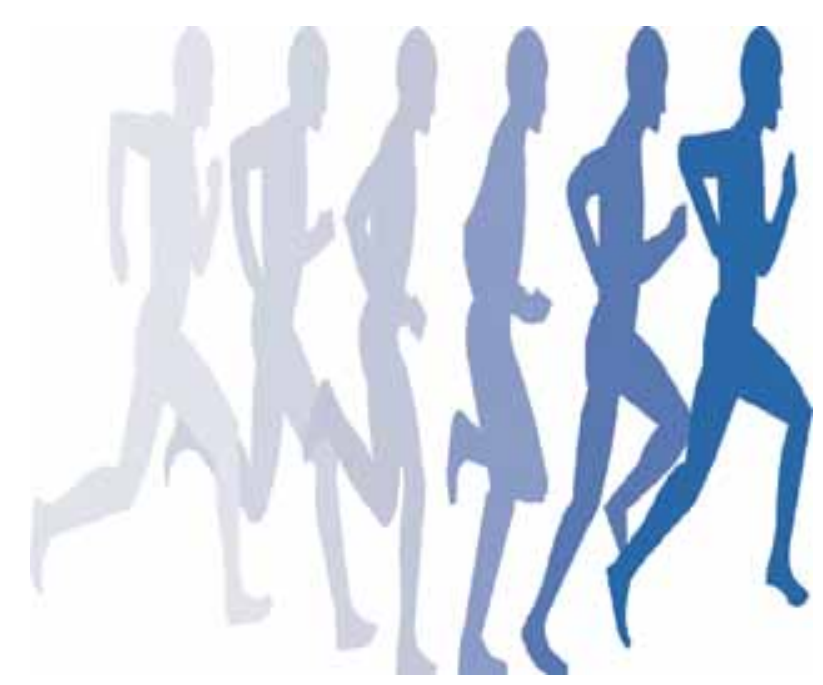

